Potential for heal th screening using I ong-term car di ovascul ar par amet ers measured by fi nger vol ume- osci I I omet ry: Pi I ot compar at i ve eval uat i on i $n$ regul ar and sl eep-depri ved activities

\begin{tabular}{|l|l|}
\hline 著者 & $\begin{array}{l}\text { Yanakoshi Takehi ro, Nat sumur a Kent a, Rol fe } \\
\text { Pet er, Hanaki Shot a, I kar ashi Aki ra, Lee } \\
\text { i hyoung, Yanakoshi Ken- i chi }\end{array}$ \\
\hline $\begin{array}{l}\text { j our nal or } \\
\text { publ i cat i on t i t l e }\end{array}$ & $\begin{array}{l}\text { I EEE Jour nal of Bi onedi cal and Heal th } \\
\text { I nf or nat i cs }\end{array}$ \\
\hline vol une & 18 \\
\hline nunber & 1 \\
\hline page r ange & 28 -35 \\
\hline year & $2014-01-01$ \\
\hline URL & ht t p: //hdl . handl e. net /2297/36900 \\
\hline
\end{tabular}




\title{
Potential for Health Screening Using Long-Term Cardiovascular Parameters Measured by Finger Volume-Oscillometry: Pilot Comparative Evaluation in Regular and Sleep-Deprived Activities
}

\author{
Takehiro Yamakoshi, Member, IEEE, Kenta Matsumura, Member, IEEE, Peter Rolfe, Member, IEEE, \\ Shota Hanaki, Akira Ikarashi, Jihyoung Lee, Student Member, IEEE, and Ken-ichi Yamakoshi, \\ Member, IEEE
}

\begin{abstract}
We explored the potential of health screening based on the long-term measurement of cardiovascular parameters using the finger volume-oscillometric technique. An automated instrument made simultaneous measurements of key cardiovascular parameters, including blood pressure, pulse pressure, heart rate, normalized pulse volume as an index of $\alpha$-adrenalin-mediated sympathetic activity, and finger arterial elasticity. These were derived from finger photoplethysmographic signals during application of cuff pressure. To assess the feasibility of achieving a screening function, measurements were made in ten healthy volunteers during $\mathbf{1 0}$ days of day-to-day living (normal condition), and carried out several times at a fixed time every day. During successive 10-day measurements, a 30-hour period of total sleep deprivation was introduced as a physiological challenge (abnormal condition). A linear discriminant analysis of the data was conducted to determine whether these two conditions could be discriminated. Periodic data collection was performed rapidly and easily, and the \%-correct classifications of normal and abnormal conditions
\end{abstract}

Manuscript received March 12, 2013; revised June 16, 2013; accepted July 17, 2013. Date of publication xx xx, 2013; date of current version yy yy, 2013 This work was supported in part by the Japan Science and Technology Agency, Grant-in-Aid for Discovery of the Seeds, under Grant 07-053, and by the Ministry of Education, Science, Sports and Culture, Grant-in-Aid for Challenging Exploratory Research under Grant 23650349 and Young Scientists (A) under Grant 24680063.

T. Yamakoshi is with the School of Mechanical Engineering, College of Science and Engineering, Kanazawa University, Kakuma-machi, Kanazawa, Ishikawa 920-1192 Japan (e-mail: takey@ staff.kanazawa-u.ac.jp).

K. Matsumura and K. Yamakoshi are with the School of Mechanical Engineering, College of Science and Engineering, Kanazawa University, Kakuma-machi, Kanazawa, Ishikawa 920-1192 Japan (e-mail: kenta16moon @ se.kanazawa-u.ac.jp; kyama@staff.kanazawa-u.ac.jp).

P. Rolfe is with the Oxford BioHorizons Ltd., 31-33 Albion Place, Maidstone ME14 5DZ UK and the Department of Automatic Measurement and Control, Harbin Institute of Technology, 92 West Dazhi Street, Nan Gang District, Harbin 150001 China (e-mail: PeterRolfe@aol.com).

S. Hanaki and J. Lee are with the Graduate School of Natural Science and Technology, Kanazawa University, Kakuma-machi, Kanazawa, Ishikawa 920-1192 Japan (e-mail: shanaki@gmail.com; jihyoung@ stu.kanazawa-u.ac. jp).

A. Ikarashi is with the Department of Medical Engineering, Aino University, 4-5-4 Higashioota, Ibaraki-shi, Osaka 567-0012 Japan (e-mail: a-ikarashi@me-u.aino.ac.jp). were $78.2 \%$ and $77.5 \%$, respectively. This ability of the method to discriminate between regular and sleep-deprived activities demonstrates its potential for healthcare screening during day-to-day living. Further investigations using larger age and gender groups of subjects including patients with cardiovascular diseases under real-life situations are required.

Index Terms-blood pressure, finger-artery elasticity index, pulse pressure, heart rate, normalized pulse volume, healthcare.

\section{INTRODUCTION}

A CADEMIC researchers and device companies are now actively developing compact, low-cost technologies for convenient and effective health screening, monitoring and personalized healthcare [1]-[3]. A recognized goal is to produce devices and the associated processing, transmission and data analysis tools capable of being integrated into the normal daily lives of millions of citizens across the world. Such technologies are being developed to allow for the early detection and therapeutic monitoring of infections, inflammatory and malignant diseases, and cardiovascular, neurological and metabolic disorders. Specific developments include point-of-care blood analyzers [4], point-of-care testing of physiological variables using a smartphone [1], [2], body area sensor networks [5] and non-intrusive physiological monitoring instruments [6], [7]. In all cases the roles of information technologies are critical.

Of relevance to this major medical and societal problem are the continued advances in the monitoring and diagnostic technologies that allow physiological measurements in ambulatory subjects and during normal daily life [1], [8]-[11]. These advances could be used to develop health screening instrumentation. It is therefore pertinent and timely to consider how such measurement technologies may be further developed and applied to achieve convenient screening for personalized healthcare.

We have previously described the design of a compact and 


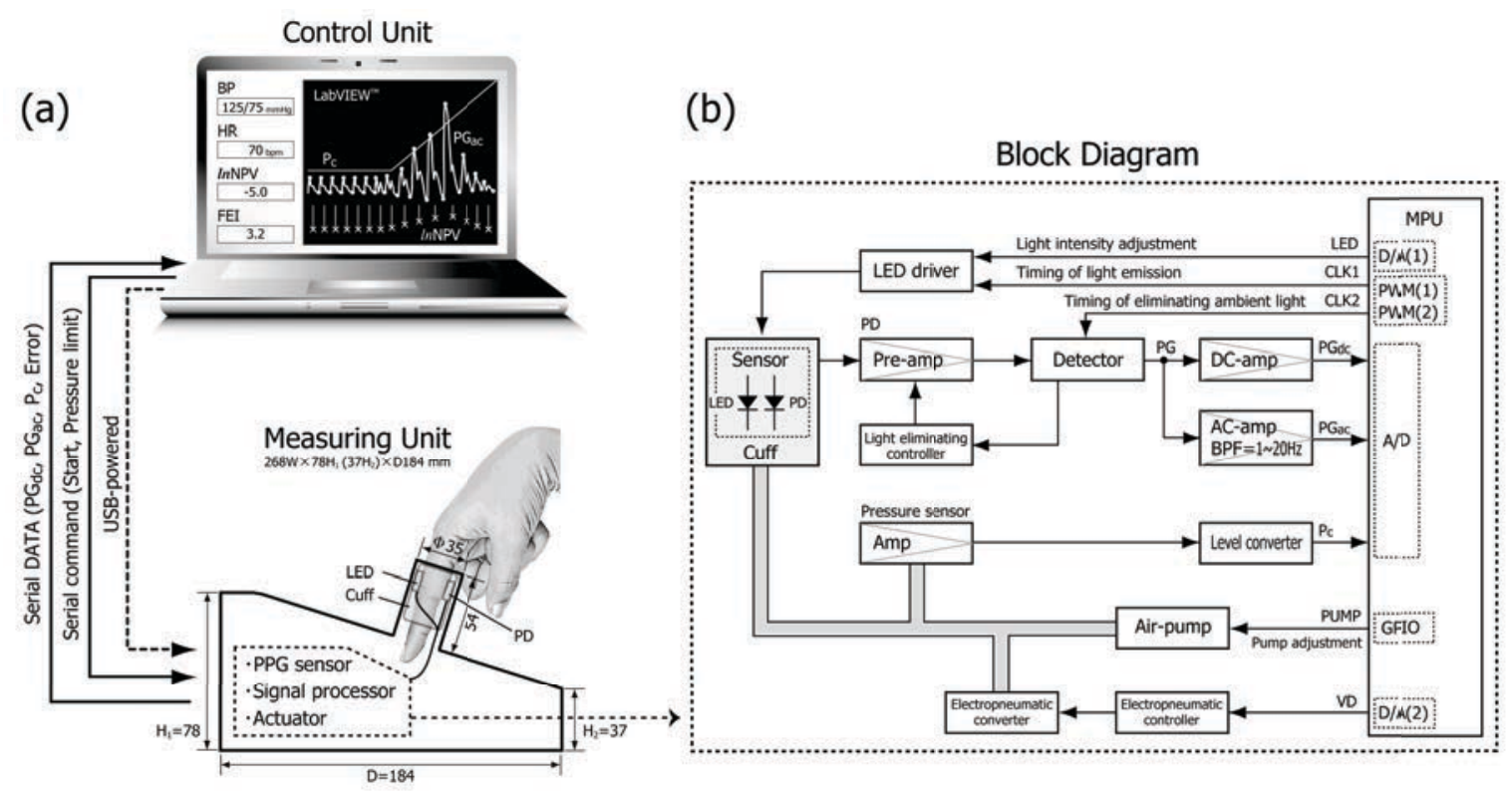

Fig. 1. Outline of the prototype system (a), and schematic block diagram of the measuring unit (b). $B P=$ blood pressure, $H R=$ heart rate, $\ln N P V=\ln$ normalized pulse volume, $F E I=$ finger-artery elasticity index, $P P G=$ photo-plethysmogram, $P G_{\mathrm{dc}}=$ direct-current component of $P P G, P G_{\mathrm{ac}}=$ alternating-current component of PPG, $P_{\mathrm{c}}=$ cuff pressure, $L E D=$ light emitting diode, $P D=$ photo diode.

convenient instrument, which accurately (compared to the conventinal method) and precisely (reproducibly) measures certain cardiovascular indices with established methods [12]. Briefly, measurements are taken from a single finger, using photo-plethysmography for the detection of peripheral pulse volume signals. The instrument then calculates: 1) systolic, mean and diastolic blood pressure (SBP, MBP and DBP, respectively) using the volume-oscillometric method [13] -[15]; 2) pulse pressure (PP), which is a strong predictor of increased large artery stiffness and high cardiovascular risk [16]; 3) heart rate (HR); 4) normalized pulse volume (NPV), which reflects $\alpha$-adrenalin-mediated sympathetic control of the finger arteriolar vessels [17], [18]; and 5) the finger-artery elasticity index (FEI) derived from a simple descriptive model of the exponential pressure-volume function for the finger vascular bed [17], [19], modified by the volume elastic modulus [20].

These cardiovascular indices are simultaneously obtained using the present instrument and, whilst each is of interest and significant in itself, the adoption of a comprehensive method for handling the combined datasets is necessary if they are to be of practical use. For example, the detection of measurements falling outside of their normal range, followed by a sequence of appropriate actions should be achieved automatically by efficient processing, transmission and storage technologies. The development of such systems to achieve a screening function that might lead in the long term to improvements in health-related disorders is a significant aim of our on-going studies. Meanwhile, the screening method for heart diseases and sleep apnea syndrome using a single physiological index, e.g., blood pressure or heart rate variability, has been reported [21]-[23]. However, to the best of our knowledge, there are no published studies concerning a comprehensive method for handling combined multiple physiological variables, and such studies are desirable to screen for some specific health conditions, including cardiovascular abnormalities, at an early stage through general daily healthcare. Therefore, we describe here an early pilot assessment of a simplified method for discriminating between regular and irregular daily activities in healthy young male volunteers, performed over 10-day periods, firstly during normal daily living and secondly with the imposition of a 30-hr period of sleep deprivation as a physiological challenge intended to create some cardiovascular effects. Discriminant analysis is then used to determine whether the method could distinguish between normal and abnormal health conditions.

\section{MATERIALS AND METHODS}

\section{A. System Description}

Fig. 1 shows an outline of the prototype system for the measurement of BP, PP, HR, NPV and FEI, part of which was reported in a preliminary publication in Japanese [12]. Briefly, the developed system comprises two main units [see Fig. 1(a)]: 1) a control unit (laptop computer, vostro1200; Dell Inc.; Kawasaki; Japan); 2) a measuring unit [268W $\times 78 \mathrm{H}_{1}$ $\left.\left(37 \mathrm{H}_{2}\right) \times 184 \mathrm{D} \mathrm{mm}\right]$ comprising a finger cuff with a cylindrical jig $(\varphi 35 \times 54 \mathrm{H} \mathrm{mm})$, a near-infrared light emitting diode (LED: SMC810; peak emission wavelength $810 \mathrm{~nm}$; Epitex Inc.; Kyoto; Japan) as a light source and a photo diode (PD: BPW34FAS; OSRAM GmbH; Munich; Germany) as a detector, an air pump, a pressure sensor and associated components. In the measuring unit, the LED is driven in a pulsatile mode at a frequency of $300 \mathrm{~Hz}$ [see circuit block diagram, Fig 1(b)]. The signal from the photodiode detector is 
first amplified by a pre-amplifier and then conditioned to subtract ambient light. The resulting signal constitutes the photo-plethysmogram (PPG), comprising of a direct-current (dc) component $\left(\mathrm{PG}_{\mathrm{dc}}\right)$ and an alternating-current (ac) component $\left(\mathrm{PG}_{\mathrm{ac}}\right)$, the latter representing the pulsatile arterial blood volume, or pulse. The dc and ac components of the PPG are further amplified. The ac amplifier has a bandpass frequency characteristic of $1-20 \mathrm{~Hz}(-3 \mathrm{~dB})$. The analogue signals $\mathrm{PG}_{\mathrm{dc}}$ and $\mathrm{PG}_{\mathrm{ac}}$, together with a signal representing the cuff pressure $\left(\mathrm{P}_{\mathrm{c}}\right)$ are sampled at a frequency of $100 \mathrm{~Hz}$ with a resolution of 12 bits and sent to the the digital data then sent to the control unit via a USB2.0 connection. The signals are subsequently displayed in real-time and calculations based on the principles of each measurement are carried out using LabVIEW2009 software (National Instruments Japan Corp., Tokyo, Japan).

The measurement process, which is started with a serial command from the control unit, has two phases, as shown in Fig. 2. Firstly, $P_{c}$ is maintained at $30 \mathrm{mmHg}$ for 10 -s to measure the NPV and HR (see Step 1 in Fig. 2); secondly, whilst applying a gradual change $(5 \mathrm{mmHg} / \mathrm{s})$ in counter-pressure, the BP, PP and FEI are measured by application of the volume-oscillometric procedure (see Step 2 in Fig. 2).

\section{B. Measurement Principle}

Normalized pulse volume (NPV), shown to be a valid index of $\alpha$-adrenalin-mediated sympathetic activity [18], [24], was calculated on a beat-by-beat basis by dividing the $\mathrm{PG}_{\mathrm{ac}}$ by $\mathrm{PG}_{\mathrm{dc}}$ on the condition that the counter-pressure is constant [17], [18], [24], giving the following equation:

$$
N P V=P G_{a c} / P G_{d c}
$$

In this study, after applying a logarithmic transformation to standardize the distribution, the $\ln \mathrm{NPV}$ was averaged over 10-s. Heart rate (HR) was derived from the peak-to-peak intervals of the $\mathrm{PG}_{\mathrm{ac}}$ signal for the same duration as for the NPV measurement. This NPV value obtained with the current instrument constitute reference data in itself, because we applied the established methods in our present instrument.

Systolic and mean blood pressure (SBP and MBP, respectively) were measured from the right index finger artery using the volume-oscillometric method [13]-[15]. The volume-oscillometric method is based on the nonlinear nature of the pressure-volume characteristics in the artery. When applying a gradual change in counter-pressure externally to the anatomical segment, characteristic changes in the amplitude of arterial volume pulsations produced by the pulse pressure are observed due to the arterial tube law [14], [15]. The determination of SBP and MBP by this method is given as the $P_{c}$ values corresponding to the systolic end point and the point of maximum amplitude of volume pulsation, respectively (see Fig. 2). Diastolic blood pressure (DBP) cannot be obtained by this method, but it can be calculated from the SBP and MBP determined above along with the volume determinant factor measured from the volume pulsation waveform [25]. Pulse pressure (PP) is calculated by

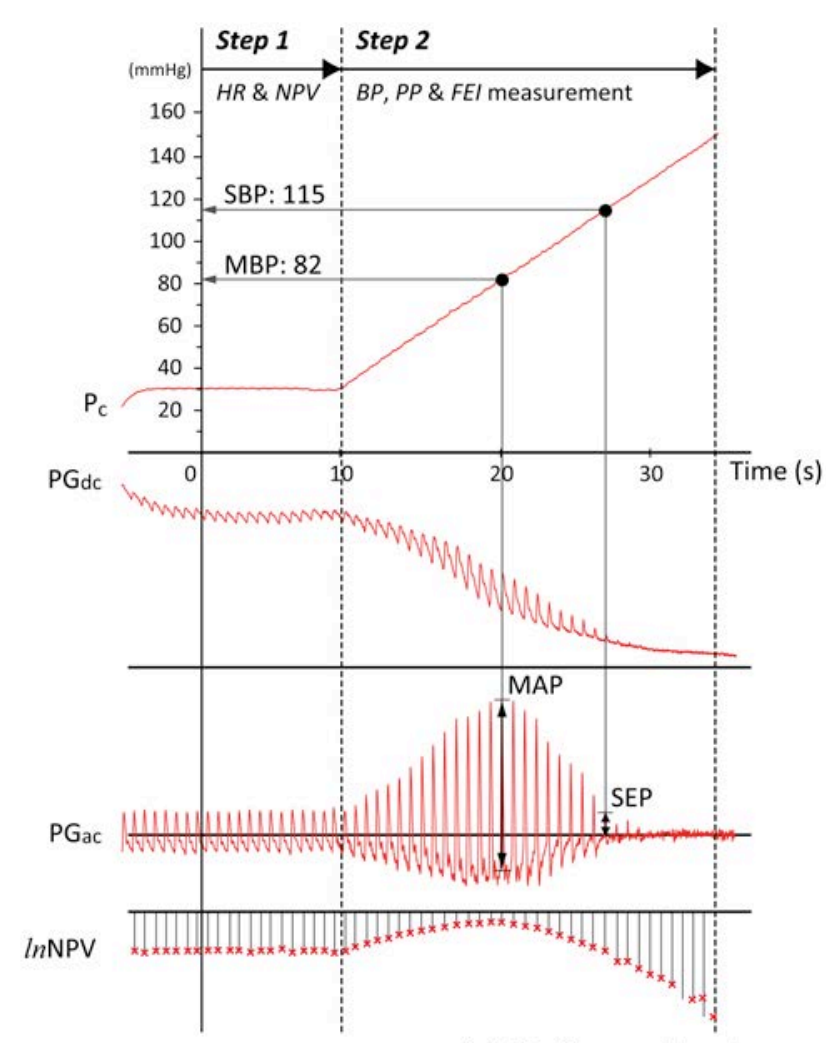

Sub.05, 21-year-old male

Fig. 2. Typical recordings of applied cuff pressure $\left(\mathrm{P}_{\mathrm{c}}\right)$, photo-plethysmographic pulsation signal $\left(\mathrm{PG}_{\mathrm{ac}} \& \mathrm{PG}_{\mathrm{dc}}\right)$, and calculated beat-by-beat $\ln \mathrm{NPV}$. $S B P=$ systolic blood pressure, $M B P=$ mean blood pressure, $P P=$ pulse pressure, $H R=$ heart rate, $\ln N P V=\ln$ normalized pulse volume, $F E I=$ finger-artery elasticity index, $M A P=$ point of maximum amplitude, $S E P=$ systolic end point.

subtracting DBP from SBP. This finger volume-oscillometric method has previously been clinically validated against direct (Oxford) and conventional upper arm-cuff methods using normotensive and hypertensive subjects [26], [27]. In addition, we have already compared BP values obtained with the present instrument to those obtained with the conventional upper arm-cuff method in a previous report (in Japanese) [12]. According to these results, the fixed bias and precision of SBP, MBP and DBP derived from Bland-Altman plots of data from the developed system and the upper arm-cuff method averaged, with a mean \pm standard deviation (data $n=625$ ), $-5.4 \pm 7.0$ $\mathrm{mmHg},-2.5 \pm 9.0 \mathrm{mmHg}$ and $-1.1 \pm 9.7 \mathrm{mmHg}$, respectively. These results clearly indicated that BP in the finger artery could be measured with acceptable accuracy using the present instrument.

Finger-artery elasticity index (FEI), shown to be a valid index of finger vascular elasticity [17], [19], and measured by the volume-oscillometric procedure mentioned above, was defined as the absolute value of the slope that follows the curvilinearity in the pressure-volume function of the finger artery, and can be calculated from the linear relationship of the log-transformed compliance index $(\mathrm{CI}:=N P V / P P)$ to the transmural pressure $\left(\mathrm{P}_{\mathrm{tr}}:=M B P-P_{c}\right)$, giving the following equation: 


$$
F E I=d(\ln C l) / d P_{t r}
$$

This FEI value obtained with the current instrument constitute reference data in itself, because we applied the established methods in our present instrument.

\section{Experimental Method}

1) Participants: Ten healthy young male participants, with a mean \pm standard deviation age, height, weight and body mass index of $21.8 \pm 0.8$ years, $169.9 \pm 4.0 \mathrm{~cm}, 59.0 \pm 6.5 \mathrm{~kg}$ and $20.4 \pm 1.6$, respectively, and no known cardiovascular disorders participated in the preliminary study. All subjects agreed to take part in the study voluntarily and signed an informed consent statement. The study was approved by the ethics commission of the Faculty of Medicine of Kanazawa University, and was performed in accordance with the ethical standards laid down in the 1964 Declaration of Helsinki and its later amendments.

2) Apparatus: The experiment was conducted in a quiet experimental room at a temperature of approximately $25^{\circ} \mathrm{C}$, which was constructed at Kanazawa University. A desk and a comfortable chair for participants to sit on were made available in this room. The prototype was placed on the right side of the participant and kept at heart level.

3) Experimental Design: The study had an ABA (A: regular activity, B: irregular activity) design. The participants were asked to maintain their regular daily routines and sleeping/waking hours from at least 2 days before commencement of the experiment, and continuing for the 10-day experimental (ABA) period, which included a 3-day rest period immediately after the B-period to avoid the possibility of influencing the following A-period.

A-period: The participants were requested to sit down on the seat. One-shot measurements (maximum 1-min) were made after a 3-min rest period and were repeated throughout the morning, afternoon and early-evening during the experimental period (3 times/day) for every participant, and were always made a significant time before main meals. B-period: The participants were asked to undergo a 30-hr period of total sleep deprivation, from day 3 through day 4 , as a physiological challenge. The reason for applying this sleep deprivation challenge was to create physiological responses and it was anticipated that the cardiovascular variables simultaneously obtained from the present instrument would reflect such responses. During, and only during, this

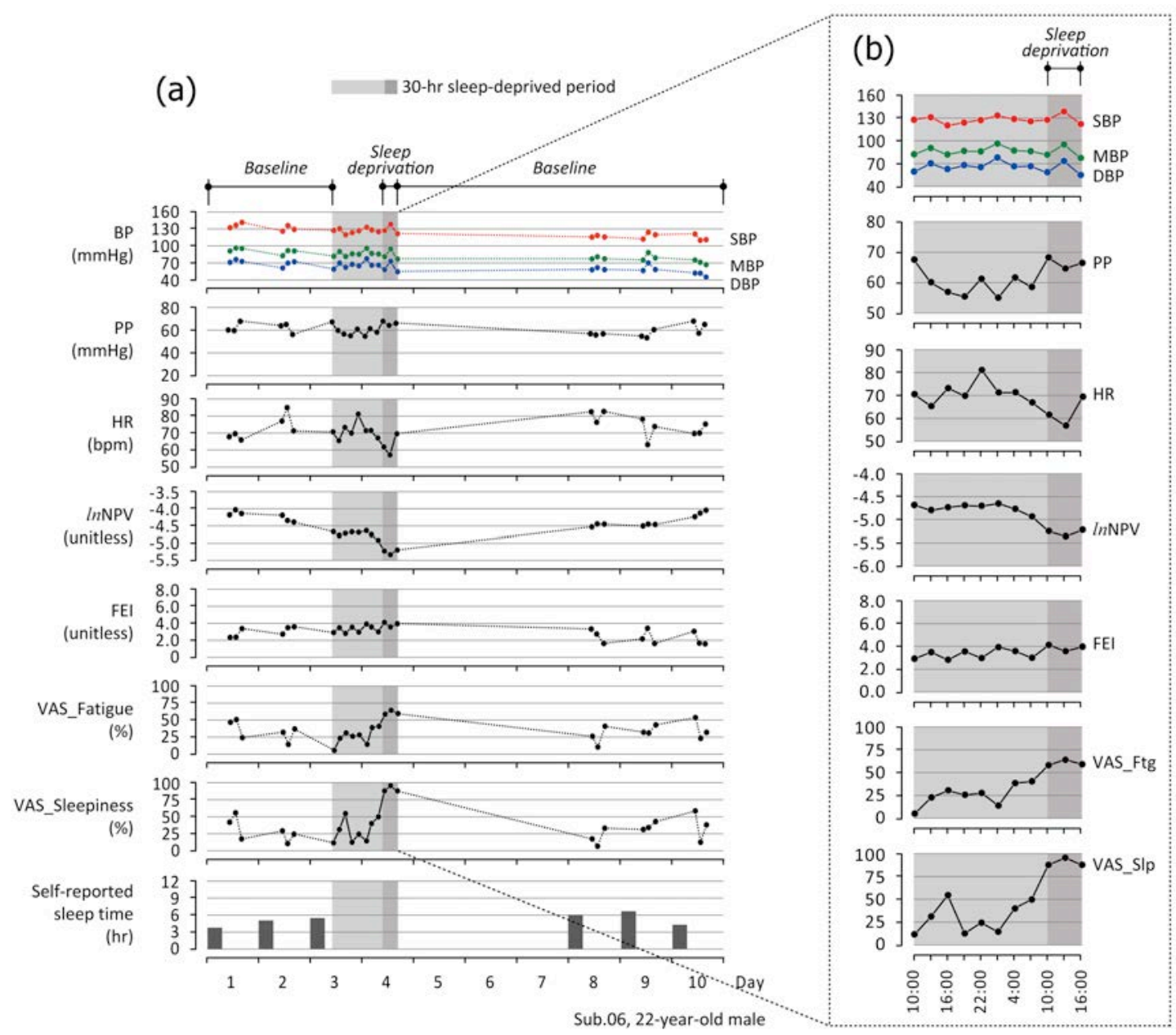

Fig. 3. Typical recordings of systolic, mean and diastolic blood pressure (SBP, MBP and DBP), pulse pressure (PP), heart rate (HR), log-transformed normalized pulse volume $(\operatorname{lnNPV})$, and finger-artery elasticity index (FEI) measured by the prototype system, the visual analogue scale (VAS) of fatigue and sleepiness, and self-reported sleep time during day-to-day living (a); and recordings of an expanded time-scale during sleep deprivation (b). 
TABLE I

SUMMARY OF LINEAR DISCRIMINANT ANALYSIS IN ALL SUBJECTS

\begin{tabular}{|c|c|c|c|c|c|c|c|c|c|c|}
\hline \multicolumn{5}{|c|}{ Linear discriminant function } & \multicolumn{6}{|c|}{ Result } \\
\hline \multirow{2}{*}{ Indices } & \multirow{2}{*}{ Coefficient } & \multirow{2}{*}{$\begin{array}{c}\text { Standardized } \\
\text { coefficient }\end{array}$} & \multirow{2}{*}{$F_{1,254}$} & \multirow{2}{*}{$p$-value } & & & \multicolumn{2}{|c|}{ Predicted } & \multirow{2}{*}{\multicolumn{2}{|c|}{$\begin{array}{l}\% \text {-Correct } \\
\text { classification }\end{array}$}} \\
\hline & & & & & & & Baseline & Sleep deprivation & & \\
\hline MBP & 0.0064 & 0.0604 & 0.22 & n.s. & \multirow{4}{*}{$\begin{array}{l}\vec{D} \\
\vec{D}_{0} \\
00 \\
0\end{array}$} & $\cong$ & \multirow{2}{*}{172} & \multirow{2}{*}{48} & $\stackrel{?}{\overrightarrow{0}}$ & \multirow{2}{*}{$78.2 \%$} \\
\hline $\mathrm{PP}$ & -0.0063 & -0.0712 & 0.34 & n.s. & & $\ddot{ص}$ & & & 品 & \\
\hline HR & 0.1019 & 0.8148 & 55.48 & $<.01$ & & \multirow{2}{*}{ 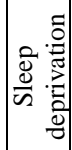 } & \multirow{2}{*}{9} & \multirow{2}{*}{31} & \multirow{2}{*}{ 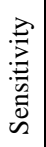 } & \multirow{2}{*}{$77.5 \%$} \\
\hline $\ln \mathrm{NPV}$ & 0.0339 & 0.5574 & 18.32 & $<.01$ & & & & & & \\
\hline FEI & -0.0101 & -0.3795 & 7.73 & $<.01$ & \multirow{2}{*}{\multicolumn{2}{|c|}{ 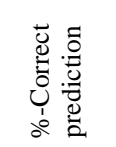 }} & $\begin{array}{c}\text { Negative predictive } \\
\text { value }\end{array}$ & $\begin{array}{c}\text { Positive predictive } \\
\text { value }\end{array}$ & & \\
\hline Constant & 0.2676 & - & - & - & & & $95.0 \%$ & $39.2 \%$ & & \\
\hline
\end{tabular}

Note. $M B P=$ mean blood pressure, $P P=$ pulse pressure, $H R=$ heart rate, $\ln N P V=\ln$ normalized pulse volume, $F E I=$ finger-artery elasticity index.

awakening B-period, using a prepared timetable referred to as Vaara's method [28], the measurement was iteratively carried out every 3 -hr from day 3 at 10:00 to day 4 at 16:00. In addition, the participants completed a questionnaire for the subjective rating of fatigue and sleepiness using a visual analogue scale (VAS; [29]) for every measurement.

4) Data Reduction and Analyses: To evaluate whether or not our system has the potential to screen for adverse effects on health, a linear discriminant analysis using the cardiovascular indices was conducted. We used MBP as a representative BP value in this analysis. We classified the collected data as after sleep deprivation and baseline; we defined sleep deprivation as the data gathered on day 4 at 07:00 (20-hr), 10:00 (24-hr), 13:00 (27-hr) and 16:00 (30-hr). Baseline is the rest of the data except for the balance of data during the 30-hr of total sleep deprivation. In addition, a linear discriminant analysis was conducted for total data. To exclude individual differences in the absolute value, the delta change $(\Delta)$ in reactivity was calculated by subtracting averaged baseline values at 10:00 from each value. The analysis was carried out using IBM SPSS Statistics 21 for Windows (IBM Inc., Tokyo, Japan).

\section{RESULTS}

Fig. 3(a) is a typical example of a 10-day trend chart from one subject, showing SBP, MBP, DBP, PP, HR, $\ln \mathrm{NPV}$ and FEI together with VAS data for fatigue and sleepiness, and the self-reported sleep time. Fig. 3(b) shows an expanded time-scale during the sleep deprivation period. The prototype instrument allowed the periodic collection of physiological data without any failed measurements. It can be seen that the variables changed depending on the physical status as judged by the VAS. In particular, it was clearly demonstrated that the $\mathrm{BP}, \mathrm{HR}$ and $\ln \mathrm{NPV}$ values varied during the period of $30-\mathrm{hr}$ sleep deprivation as compared with the values during the rest period.

We classified the dataset into baseline and sleep deprivation groups [see the top of Fig. 3(a)]. Table I shows the summary of the linear discriminant analysis for all subjects combined. The classification function and its results are shown in the left and right parts, respectively. Based on the $p$-values, the effective indices for discrimination were found to be $\mathrm{HR}\left(F_{1}\right.$, $\left.{ }_{254}=55.48, p<0.01\right), \ln \mathrm{NPV}\left(F_{1,254}=18.32, p<0.01\right)$ and FEI $\left(F_{1,254}=7.73, p<0.01\right)$. The $\%$-correct classifications of baseline (i.e., specificity) and sleep deprivation (i.e., sensitivity) were $78.2 \%$ and $77.5 \%$, respectively, and the $\%$-correct predictions of baseline (i.e., negative predictive value) and sleep deprivation (i.e., positive predictive value) were $95.0 \%$ and $39.2 \%$, respectively.

\section{DISCUSSION}

A simplified method ultimately intended for health screening, based on the non-invasive simultaneous measurement of SBP, MBP, DBP, PP, HR, NPV and FEI using a finger cuff, was found to perform reliably in preliminary tests with ten healthy young male volunteers over 10 days of normal day-to-day living. The method was found to be able to discriminate between the normal status and an abnormal condition created by a 30 -hr period of total sleep deprivation.

It has been reported that 30-hr sleep deprivation changes adjustments in the autonomic regulation of the heart, i.e., it decreases heart rate [30], [31]. Taking the effective indices obtained here into consideration, our findings are consistent with these reports. A decrease in $\ln \mathrm{NPV}$, representing peripheral vasoconstriction, could imply compensatory behavior to regulate BP in response to a drop in HR. Such physiological reactions are plausible. Thus, the percentages of 
correctly classified samples (baseline: 78.2\%, sleep deprivation: $77.5 \%$ ) might be considered to be highly satisfactory.

The cardiovascular variables we used in the analysis were MBP, PP, HR, $\ln$ NPV and FEI, which were the key variables obtained from this prototype instrument. It has been reported that the FEI reflects structural (not functional) changes in the finger artery [17], [19], and the PP is also known to change with age following the progressive stiffening of larger arteries [16]. For these reasons, FEI and PP are generally recognized as long-term cardiovascular risk factors. Concerning FEI, our present results show that this index was effective for discriminating between normal and the imposed acute sleep deprivation condition, as shown in Table I. This is, in fact, inconsistent with the previous findings mentioned above. Although a definite reason for this is not yet clear, one possibility could be considered. According to Tanaka et al.'s measurement procedure [19], FEI is calculated from the average value of eight or nine measurements. Therefore, the one-shot measurement applied in the present prototype might be inadequate to evaluate the long-term elasticity of small arteries. However, if we use day-to-day values in an appropriate database, we could estimate the long-term finger artery stiffness by calculating the moving-average of these accumulated data. As for the PP measurements in our present results, these were found to be ineffective indices for discriminating between the normal and the sleep deprivation conditions. Although this result was to be expected in the present study, it is probably more appropriate to process the data in the same way as for FEI. In any case, it seems reasonable to speculate that our system, by using the measured FEI and PP individually, might be able to screen for the development of cardiovascular diseases if data collected over the medium to long term are used for the discriminant analysis; this needs to be validated adequately by recruiting patients with cardiovascular diseases in a future study.

By contrast, abnormalities of health occurring in the short-term, e.g., events of acute stress, will particularly be predicted by the $\mathrm{BP}, \mathrm{HR}$ and/or $\ln \mathrm{NPV}$ indices, as demonstrated here in the sleep deprivation example. Naturally, the indices that act as effective predictors of abnormality will be different for different health conditions, e.g., physical and/or mental exhaustion, degradation of general health with aging, or a cardiac disease. It is likely that there will also be differences in the most effective predictor among individuals for any given health condition. Methodological development to achieve the most appropriate discriminant function for each condition and the associated information and communication technology to create a complete health screening system is therefore required. To achieve this, further investigations will be needed in larger age and gender groups of subjects with a variety of health conditions under real-life situations to develop and test the system for health screening.

Further physiological variables could be added in our system, and we are investigating blood constituent measurements, e.g., the levels of blood glucose, albumin, cholesterol and alcohol, using multiple wavelength photo-plethysmography to assist with general daily healthcare. This work is still in its infancy [32]-[35], but future progress is expected, and these are targets to be integrated eventually into our system. In connection with this, blood alcohol measurement using the optical principle is interesting and significant. Our method is intended to be a simpler technique that could lead to the development of instrumentation for use by motor vehicle drivers in order to reduce health and alcohol related motor vehicle accidents as novel vehicle safety locks.

This was an early pilot trial and there were therefore several possible limitations. Firstly, only ten healthy young men participated in the study. Studies with larger groups, with a wider age range, including both male and female subjects and patients (e.g., with cardiovascular disease), need to be carried out. Secondly, in this first pilot study we used the sleep deprivation challenge to simulate an abnormal health condition in the laboratory environment and it will now be necessary to examine a variety of common health conditions under real-life constraints in order to test the suitability of this method for health screening more comprehensively. Finally, we only used discrete-time measurements of cardiac phenomena for acute stress in this preliminary study. Monthly or yearly long-term monitoring, if possible by applying chronic stress, in healthy participants or outpatients with cardiovascular diseases should be conducted in a future study. In this manner, many technical, theoretical and experimental challenges still need to be overcome in order to improve the prevention and treatment of cardiovascular diseases that may be caused by the events of chronic and acute stress. The preliminary trial of our simplified method with a prototype instrument in healthy young adults could open up new possibilities for the design of convenient systems for cardiovascular health screening.

\section{CONCLUSION}

A simplified method for health screening based on non-invasive cardiovascular measurements from just one finger using the volume-oscillometric technique and its preliminary assessment are described. Assessment of the method for screening regular and irregular daily activities using the cardiovascular parameters was conducted for ten healthy volunteers during 10 days of day-to-day living. The method was able to discriminate between the normal status and an abnormal condition created by a $30-\mathrm{hr}$ period of total sleep deprivation as a physiological challenge. This ability of the method to discriminate between regular and sleep-deprived activities demonstrates its potential for healthcare screening during day-to-day living. Further investigations using larger age and gender groups of subjects including patients with cardiovascular diseases under real-life situations are required.

\section{ACKNOWLEDGMENT}

The authors would like to give their sincere thanks to Mr. Hideaki Konishi, Kanazawa University, for his assistance with the experiment. 


\section{REFERENCES}

[1] K. Matsumura and T. Yamakoshi, "iPhysioMeter: A new approach for measuring heart rate and normalized pulse volume using only a smartphone," Behavior Research Methods, Feb, 2013. (Published Online First)

[2] Y. G. Lee, W. S. Jeong, and G. Yoon, "Smartphone-based mobile health monitoring," Telemedicine Journal and e-Health, vol. 18, no. 8, pp. 585-90, Oct, 2012

[3] P. Klasnja and W. Pratt, "Healthcare in the pocket: mapping the space of mobile-phone health interventions," Journal of Biomedical Informatics, vol. 45, no. 1, pp. 184-98, Feb, 2012.

[4] F. Olasagasti and J. C. Ruiz de Gordoa, "Miniaturized technology for protein and nucleic acid point-of-care testing," Translational Research, vol. 160, no. 5, pp. 332-45, Nov, 2012.

[5] S. Ullah, H. Higgins, B. Braem, B. Latre, C. Blondia, I. Moerman, S. Saleem, Z. Rahman, and K. S. Kwak, "A comprehensive survey of Wireless Body Area Networks : on PHY, MAC, and Network layers solutions," Journal of Medical Systems, vol. 36, no. 3, pp. 1065-94, Jun, 2012.

[6] K. Motoi, M. Ogawa, T. Yamakoshi, and K. Yamakoshi, "Fusion physiological sensing system for healthcare," Technological Advancements in Biomedicine for Healthcare Applications, J. Wu, ed., pp. 298-313, Pennsylvania: IGI Global, 2012.

[7] K. Yamakoshi, "Current status of noninvasive bioinstrumentation for healthcare," Sensors and Materials, vol. 23, no. 1, pp. 1-20, Jan, 2011.

[8] M. Z. Poh, D. J. McDuff, and R. W. Picard, "Advancements in noncontact, multiparameter physiological measurements using a webcam," IEEE Transactions on Biomedical Engineering, vol. 58, no. 1, pp. 7-11, Jan, 2011.

[9] S. Altunkan, K. Oztas, and B. Seref, "Arterial stiffness index as a screening test for cardiovascular risk: a comparative study between coronary artery calcification determined by electron beam tomography and arterial stiffness index determined by a VitalVision device in asymptomatic subjects," European Journal of Internal Medicine, vol. 16, no. 8, pp. 580-4, Dec, 2005.

[10] M. Y. Cortez-Cooper, J. A. Supak, and H. Tanaka, "A new device for automatic measurements of arterial stiffness and ankle-brachial index," The American Journal of Cardiology, vol. 91, no. 12, pp. 1519-22, A9, Jun, 2003.

[11] K. Yamakoshi, and A. Kamiya, "Noninvasive measurement of arterial blood pressure and elastic properties using photoelectric plethysmography technique," Medical Progress Through Technology, vol. 12, no. 1-2, pp. 123-43, Jan, 1987.

[12] T. Yamakoshi, K. Matsumura, S. Hanaki, A. Ikarashi, P. Rolfe, J. H. Lee Y. Yamakoshi, H. Hirose, and K. Yamakoshi, "[Development of a novel system based on the simultaneous measurement of physiological variables for safer driving and general daily healthcare]," Transactions of Japanese Society for Medical and Biological Engineering, vol. 50, no. 2, pp. 227-36, May, 2012. (in Japanese)

[13] K. Yamakoshi, "Non-invasive cardiovascular haemodynamic measurements," Sensors in Medicine and Health Care (Sensors Applications, Volume 3), P. A. Oberg, T. Togawa and F. Spelman, eds., pp. 107-60, Weinheim: Wiley-VCH Verlag, 2004.

[14] K. Yamakoshi, H. Shimazu, M. Shibata, and A. Kamiya, "New oscillometric method for indirect measurement of systolic and mean arterial pressure in the human finger. Part 1: Model experiment," Medical \& Biological Engineering \& Computing, vol. 20, no. 3, pp. 307-13, May, 1982.

[15] K. Yamakoshi, H. Shimazu, M. Shibata, and A. Kamiya, "New oscillometric method for indirect measurement of systolic and mean arterial pressure in the human finger. Part 2: Correlation study," Medical \& Biological Engineering \& Computing, vol. 20, no. 3, pp. 314-8, May, 1982.

[16] P. Verdecchia, G. Schillaci, C. Borgioni, A. Ciucci, S. Pede, and C. Porcellati, "Ambulatory pulse pressure: a potent predictor of total cardiovascular risk in hypertension," Hypertension, vol. 32, no. 6, pp. 983-8, Dec, 1998.

[17] G. Tanaka, Y. Sawada, K. Matsumura, K. Yamakoshi, and T. Okayasu, "Exponential model of pressure-volume relationship in the finger artery: Theoretical and experimental evaluation of vascular tone under mental stress and reactive hypermia," Advances in Psychology, S. Shohov, ed., pp. 89-109, New York: Nova Science Publishers, Inc., 2005.

[18] Y. Sawada, G. Tanaka, and K. Yamakoshi, "Normalized pulse volume (NPV) derived photo-plethysmographically as a more valid measure of the finger vascular tone," International Journal of Psychophysiology, vol. 41, no. 1, pp. 1-10, May, 2001.

[19] G. Tanaka, K. Yamakoshi, Y. Sawada, K. Matsumura, K. Maeda, Y. Kato, M. Horiguchi, and H. Ohguro, "A novel photoplethysmography technique to derive normalized arterial stiffness as a blood pressure independent measure in the finger vascular bed," Physiological Measurement, vol. 32, no. 11, pp. 1869-83, Nov, 2011.

[20] H. Shimazu, K. Yamakoshi, and A. Kamiya, "Noninvasive measurement of the volume elastic modulus in finger arteries using photoelectric plethysmography," IEEE Transactions on Biomedical Engineering, vol. 33, no. 8, pp. 795-8, Aug, 1986.

[21] A. Heitmann, T. Huebner, R. Schroeder, S. Perz, and A. Voss, "Multivariate short-term heart rate variability: a pre-diagnostic tool for screening heart disease," Medical \& Biological Engineering \& Computing, vol. 49, no. 1, pp. 41-50, Jan, 2011.

[22] M. R. Law, N. J. Wald, and J. K. Morris, "The performance of blood pressure and other cardiovascular risk factors as screening tests for ischaemic heart disease and stroke," Journal of Medical Screening, vol. 11 , no. 1, pp. 3-7, 2004.

[23] F. Roche, J. M. Gaspoz, I. Court-Fortune, P. Minini, V. Pichot, D. Duverney, F. Costes, J. R. Lacour, and J. C. Barthelemy, "Screening of obstructive sleep apnea syndrome by heart rate variability analysis," Circulation, vol. 100, no. 13, pp. 1411-5, Sep, 1999.

[24] J. H. Lee, K. Matsumura, T. Yamakoshi, P. Rolfe, N. Tanaka, K. H. Kim, and K. Yamakoshi, "Validation of normalized pulse volume in the outer ear as a simple measure of sympathetic activity using warm and cold pressor tests: towards applications in ambulatory monitoring," Physiological Measurement, vol. 34, no. 3, pp. 359-75, Mar, 2013.

[25] H. Shimazu, H. Ito, H. Kobayashi, and K. Yamakoshi, "Idea to measure diastolic arterial pressure by volume oscillometric method in human fingers," Medical \& Biological Engineering \& Computing, vol. 24, no. 5, pp. 549-54, Sep, 1986.

[26] Y. Imai, M. Nihei, K. Abe, S. Sasaki, N. Minami, M. Munakata, S. Yumita, Y. Onoda, H. Sekino, K. Yamakoshi, and et al., "A finger volume-oscillometric device for monitoring ambulatory blood pressure: laboratory and clinical evaluations," Clinical and Experimental Hypertension. Part A, Theory and Practice, vol. 9, no. 12, pp. 2001-25, 1987.

[27] Y. Imai, K. Abe, S. Sasaki, H. Sekino, K. Yamakoshi, and K. Yoshinaga, "A new automated volume-oscillometric device for monitoring ambulatory blood pressure in the human finger in man," Journal of Hypertension, vol. 4, pp. S662-4, 1986.

[28] J. Vaara, H. Kyrolainen, M. Koivu, M. Tulppo, and T. Finni, "The effect of 60-h sleep deprivation on cardiovascular regulation and body temperature," European Journal of Applied Physiology, vol. 105, no. 3, pp. 439-44, Feb, 2009.

[29] R. C. Wilson, and P. W. Jones, "A comparison of the visual analogue scale and modified Borg scale for the measurement of dyspnoea during exercise," Clinical Science, vol. 76, no. 3, pp. 277-82, Mar, 1989.

[30] A. L. Holmes, H. J. Burgess, and D. Dawson, "Effects of sleep pressure on endogenous cardiac autonomic activity and body temperature," Journal of Applied Physiology, vol. 92, no. 6, pp. 2578-84, Jun, 2002.

[31] H. I. Chen, "Effects of 30-h sleep loss on cardiorespiratory functions at rest and in exercise," Medicine and Science in Sports and Exercise, vol. 23, no. 2, pp. 193-8, Feb, 1991.

[32] T. Yamakoshi, M. Ogawa, K. Matsumura, S. Miyazaki, Y. Itasaka, T. Ida, Y. Yamakoshi, H. Hirose, and K. Yamakoshi, "[Towards non-invasive optical blood alcohol measurement: Multi-variate analysis of in vitro NIR spectra]," IEEJ Transactions on Electronics, Information and Systems, vol. 132, no. 12, pp. 2059-64, Dec, 2012. (in Japanese)

[33] T. Yamakoshi, M. Ogawa, K. Matsumura, Y. Itasaka, S. Miyazaki, Y. Yamakoshi, P. Rolfe, H. Hirose, and K. Yamakoshi, "[A preliminary study on development of a novel optical instrument for non-invasive blood alcohol measurement: Proposal of Pulse Alcometry]," Transactions of Japanese Society for Medical and Biological Engineering, vol. 50, no. 2, pp. 237-47, May, 2012. (in Japanese)

[34] M. Ogawa, T. Yamakoshi, K. Motoi, K. Nishida, Y. Yamakoshi, and K. Yamakoshi, "Determination of concentrations of glucose and human serum albumin in mixtures in phosphate-buffered solution by near-infrared spectroscopy," Sensors and Materials, vol. 24, no. 6, pp. 323-33, July 2012, 2012.

[35] K. Yamakoshi, and Y. Yamakoshi, "Pulse glucometry: A new approach for noninvasive blood glucose measurement using instantaneous differential near-infrared spectrophotometry," Journal of Biomedical Optics, vol. 11, no. 5, pp. 054028, Sep-Oct, 2006. 


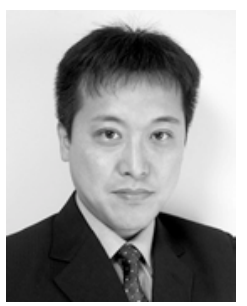

Takehiro Yamakoshi (M'06) received the B.S. and M.S. in Engineering from Muroran Institute of Technology, Japan, and the Ph.D. in Engineering from Fukui University, Japan.

$\mathrm{He}$ was an Associate Professor in the Graduate School of Natural Science and Technology, Kanazawa University, Japan, since 2006, and has been a Postdoctoral Fellow in the School of Mechanical Engineering, College of Science and Engineering at Kanazawa University, Japan, since 2012.

Dr. Yamakoshi's current research interests include physiological measurement, bio-signal analysis, biomedical optics, health informatics, and physiological measurement for safer driving.

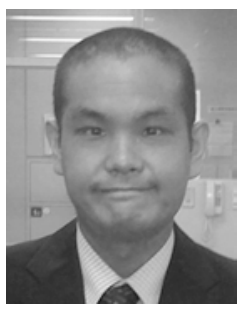

Kenta Matsumura (M'13) received the B.A. in Social Welfare from Hokusei Gakuen University, Japan, and the M.A. and Ph.D. in Human Sciences from Hokkaido University, Japan.

He was a Research Fellow of the Japan Society for the Promotion of Science from 2006 to 2008, and was a Research Fellow of the National Center of Neurology and Psychiatry from 2008 to 2011 , and has been a Postdoctoral Fellow in the School of Mechanical Engineering, College of Science and Engineering, Kanazawa University, Japan, since 2011.

Dr. Matsumura's current research interests include cardiovascular psychophysiology, cognitive neuroscience, post-traumatic stress disorder, and soft computing.

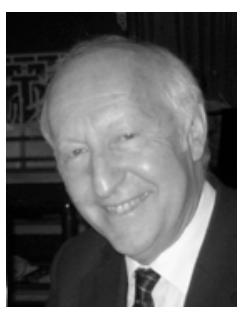

Peter Rolfe (M'88) received a Ph.D. in Biomedical Engineering from the Postgraduate Medical School, University of London.

He was founder Director of the Biomedical Engineering Centre, Oxford, the Centre/Institute Science \& Technology in Medicine, Keele, and a WHO Collaborating Centre, Oxford. He is Founder \& Director of Oxford BioHorizons Ltd, UK, and a Visiting Professor in the Department of Automatic Measurement and Control, Harbin Institute of Technology, China.

Professor Rolfe's research interests include invasive and non-invasive physiological measurement, cell/tissue engineering, near infra-red spectroscopy \& biomedical optics, micro- and nanosensors, environmental monitoring, and appropriate technology for perinatal care in developing countries.

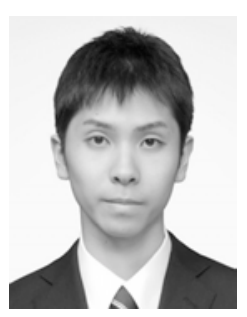

Shota Hanaki received the B.S. and M.S. in Engineering from Kanazawa University, Japan.

He was a master course student in the Graduate School of Natural Science and Technology, Kanazawa University, Japan.

Mr. Hanaki's research interests include a study examining the physiological effects of various stresses on drivers aiming at safer driving.

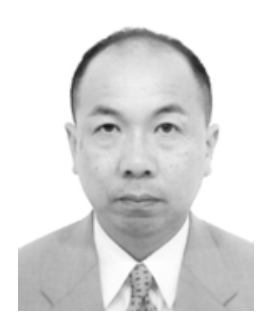

Akira Ikarashi received the B.S. in Engineering from Niigata University, Japan, and the M.S. in Engineering from Hokkaido University, Japan, and the Ph.D. in Engineering from Kanazawa University, Japan.

He is an Associate Professor in the Department of Medical Engineering, Aino University, Japan.

Dr. Ikarashi's research interests include physiological measurement, bio-signal analysis, and medical instrumentation.

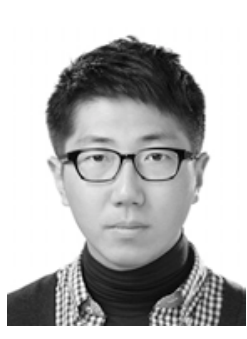

Lee Jihyoung (SM'13) received the B.S. and M.S. in Engineering from Dankook University, Korea.

$\mathrm{He}$ is a doctoral student in the Graduate School of Natural Science and Technology, Kanazawa University, Japan.

Mr. Lee is currently conducting a study examining the healthcare instrumentation. His research interests include physiological measurement, medical device, and analog circuit \& system.

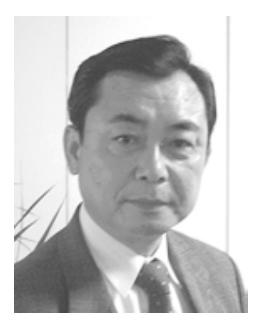

Ken-ichi Yamakoshi (M'02) received the B.S. and M.S. in Engineering from Waseda University, Japan, and the Ph.Ds. in Medicine and Engineering from Tokyo Medical and Dental University, Japan, and Waseda University, respectively.

He was a Professor in the School of Mechanical Engineering, College of Science and Engineering, Kanazawa University, Japan, and has been its Emeritus Professor, as well as a Professor in the School of Medicine, Showa University, Japan, and in the Faculty of Clinical Engineering, Hokkaido Institute of Technology, Japan, since 2013.

Professor Yamakoshi's research interests include physiological measurement, non-invasive biomedical sensors and instrumentation, and welfare engineering. 